\title{
INSATISFACCIÓN POR LA IMAGEN CORPORAL Y LA BAJA AUTOESTIMA POR LA APARIENCIA FÍSICA EN ESTUDIANTES DE LA FACULTAD DE MEDICINA DE LA UNIVERSIDAD DE LOS ANDES DEL ESTADO MERIDA VENEZUELA
}

\author{
Lic. Pedro López Atencio ${ }^{1}$, Dr. José R. Prado P. ${ }^{2}$, Lic. Mercedes Montilla ${ }^{2}$, \\ Lic. Zarela Molina Viana ${ }^{2}$, Lic. Glenda Da Silva ${ }^{3}$ y Esp. Fanny Arteaga ${ }^{4}$ \\ ${ }^{1}$ Departamento de Nutrición Social Escuela de Nutrición y Dietética, Facultad de \\ Medicina. Universidad de Los Andes.lopezp@ula.ve \\ ${ }^{2}$ Departamento de Educación Física Facultad de Humanidades y Educación, \\ Universidad de Los Andes, jrpp@ula.ve \\ ${ }^{3}$ Departamento de Nutrición y Alimentación, Escuela de Nutrición y Dietética, \\ Facultad de Medicin, Universidad de Los Andes. \\ ${ }^{4}$ Departamento de Biopatología Facultad de Odontología, Universidad de Los \\ Andes. \\ Mérida-Venezuela
}

\section{RESUMEN}

La presente investigación tuvo como propósito central evaluar la insatisfacción por la imagen corporal y su relación con la baja autoestima por la apariencia física en estudiantes de la Facultad de Medicina de la Universidad de Los Andes en Mérida Venezuela. El estudio fue de tipo no experimental correlacional. La muestra estuvo constituida por 189 estudiantes, $27 \%$ masculino y $73 \%$ femenino, con una edad promedio de 19,58 años $\pm 1,57$ (varones 19,81 años $\pm 1,74$ y para las mujeres 20,24 años $\pm 1,76)$. Los participantes fueron seleccionados de los cursos del primer año de las carreras de: medicina, enfermería y nutrición, los cuales fueron seleccionados de forma intencional. El instrumento empleado para determinar la Insatisfacción por la Imagen Corporal fue el Body Shape Questionnaire (Cooper y Taylor, 1987). El método de Graffar Modificado fue utilizado para determinar el estrato socioeconómico de los participantes (Méndez y De Méndez 1994). A los datos se les aplicó el análisis descriptivo (frecuencia, porcentajes, media) e inferencial (ANOVA de un solo factor a través del paquete estadístico SPSS versión 9.0). Entre los principales hallazgos en el estudio se pudo determinar una asociación estadísticamente significativa entre la insatisfacción con la imagen corporal, la baja autoestima por la apariencia física con el género $\chi^{2} \quad(2, N=189)=9,686, p=0,008$. A través de ANOVA se pudo determinar que las diferencias en las medias para los niveles de insatisfacción y baja autoestima con la imagen corporal y el género son estadísticamente significativas, $F=11,236 ; p=0,008, F=10,23 ; p=0,002$ respectivamente. Conclusiones: los resultados obtenidos permiten sugerir sobre la relación que existe entre la insatisfacción y la baja autoestima por la apariencia física, existiendo un rechazo por la propia imagen corporal causada por la percepción distorsionada o no de la misma, lo que podría afectar la autoestima por la apariencia física. Además, se observa que la salud psicológica de los estudiantes se relaciona más con su satisfacción por su apariencia física que como se percibe su imagen corporal. Por lo tanto, este grupo de participantes debe analizarse en relación con su autoestima por la apariencia física, como una expresión del ambiente institucional. También es importante enfatizar que el género puede ser un factor de riesgo en los desórdenes de hábitos alimenticios. Consideramos lo anterior porque las mujeres mostraron niveles de insatisfacción más altos, ya que su apariencia física se encuentra condicionada por una mayor insatisfacción con su imagen corporal tal como la perciben, que se caracteriza por una sobre estimación de la dimensión física de la imagen corporal.

PALABRAS CLAVES: baja autoestima por apariencia física, imagen corporal, insatisfacción por la imagen corporal, trastornos de la conducta alimentaria. 


\title{
MHSalud \\ URL www.una.ac.cr/mhsalud
}

\section{BODY IMAGE DISSATISFACTION AND LOW SELF-ESTEEM DUE TO PHYSICAL APPEAREANCE IN STUDENTS OF THE FACULTY OF MEDICINE AT THE UNIVERSITY OF LOS ANDES (MÉRIDA, VENEZUELA)}

\author{
ABSTRACT
}

The purpose of this investigation was to evaluate body image dissatisfaction in relation to low self-esteem due to physical appearance in students of the Faculty of Medicine at the University of Los Andes in Mérida, Venezuela. It was a nonexperimental and correlational study. The sample included 189 students (27\% male and $73 \%$ female) with an average age of $19.58 \pm 1.57$ (men: 19.81 years of age \pm 1.74 and women: 20.24 years of age \pm 1.76 ). Participants were intentionally selected from first-year courses of the Medicine, Nursing and Nutrition programs. The Body Shape Questionnaire (BSQ) (Cooper and Taylor, 1987) was the instrument used to measure body image dissatisfaction and Graffar's modified method (Méndez and De Méndez, 1994) was applied to determine the participants' socioeconomic status. A descriptive analysis (frequency, percentages, mean) and an inferential analysis (one-way ANOVA) were applied to the data using SPSS (Statistical Package for Social Sciences) version 9.0. One of the most important findings in this study was the determination of a statistically significant relationship between dissatisfaction and body image and between low self-esteem and gender $\chi^{2}(2, N=189)=$ 9.686, $p=0.008$. Using ANOVA also helped determine that differences in the mean for dissatisfaction and low self-esteem levels with body image and gender are statistically significant, $F=11.236 ; p=0.008, F=10.23 ; p=0.002$, respectively. Conclusions: results obtained suggest a relationship between dissatisfaction and low self-esteem due to physical appearance. Consequently, subjects reject their body image because of a distorted or undistorted perception of their physical appearance, which can possibly affect self-esteem. Moreover, it is observed that the students' psychological health is more related to their satisfaction with their body-image than to the way their body image is perceived. Consequently, this group of participants must be analyzed regarding their self-esteem due to body image, as an expression in the institutional environment. It is also important to emphasize that gender may be a risk factor concerning eating disorders. We believe the foregoing because women showed higher dissatisfaction levels because of their physical appearance being conditioned by a higher dissatisfaction with their perceived body image, which is characterized by an overestimation of the physical dimension of their body image.

KEY WORDS: low self-esteem due to physical appearance, body image, body-image dissatisfaction, eating disorders.

\section{INTRODUCCIÓN}

Banfield y McCabe (2002), han señalado que en los últimos años la imagen corporal ha tomado un inmenso auge en las sociedades modernas, muchas de las cuales han creado toda una subcultura basada en la percepción y la importancia de la imagen ideal. De igual forma, los referidos autores han resaltado la influencia de los medios de comunicación y la presión social entre los factores de mayor influencia para seguir una imagen ideal, que en muchas ocasiones es imposible o difícil de alcanzar.

La imagen corporal hoy constituye la base de muchos estudios desde el punto de vista sociológico en virtud de que la percepción de la misma es uno de los factores de riesgo asociado a los trastornos de la conducta alimentaria. Dichos trastornos ya no constituyen solamente un problema de salud pública en los países desarrollados, sino también en aquellos donde su perfil de salud está caracterizado por altos niveles de pobreza.

Según Brown, Cash y Milkulka (1990), la imagen corporal incluye múltiples dimensiones como las actitudes, la cognición, el comportamiento, los afectos, el miedo a ser obeso, la distorsión del cuerpo, la insatisfacción corporal, la evaluación, la preferencia por la delgadez, la restricción al comer y la percepción.

La percepción de la imagen corporal es parte integral del funcionamiento de una persona y una distorsión de la misma puede llevar o contribuir a una condición más seria, como lo son 


\section{MHSalud}

URL www.una.ac.cr/mhsalud

los Trastornos de la Conducta Alimentaria (TAC). Los más prevalentes son la anorexia nerviosa y la bulimia nerviosa (American Psychiatric Association, 2000).

En un estudio realizado por Xie y otros (2003), destacan que las personas con autopercepción de sobrepeso presentaron mayor riesgo de baja autoestima y depresión que los individuos con percepción de imagen corporal normal o de bajo peso. Así mismo, estudios realizados por Straus y Pollack (2003), evidencian que los individuos con autopercepción de sobrepeso son socialmente marginados y que la insatisfacción corporal y la discriminación pueden complicar las consecuencias sociales y emocionales.

Bonilla y Martínez (2001), mencionan que la prevalencia de los TAC ha aumentado, no sólo en el número de personas que lo padecen, sino que se hal extendido por amplios grupos poblacionales, existiendo incluso, indicios de estas patologías alimentarias bajo algunos comportamientos considerados habituales o incluso deseables, como las dietas restrictivas o el ejercicio excesivo. Un estudio realizado por Buddeberg, Klaghofer y Reed (1999), el cual tuvo como propósito explorar la asociación entre el peso corporal, desórdenes psiquiátricos e imagen corporal en féminas jóvenes, encontraron que, en general las jóvenes que presentaron sobrepeso tenían una imagen corporal negativa, es decir, se sentían menos atractivas y menos confiadas en sí mismas. También encontraron una correlación positiva entre diagnósticos psiquiátricos y una visión no favorable de la imagen corporal. Asimismo, mayores distorsiones en la imagen corporal y mayor sobrepeso, se correlacionaron con mayor morbilidad psiquiátrica.

De este modo, mejorando la satisfacción corporal no sólo permitirá influir sobre la incidencia de los trastornos del comportamiento alimentario, sino también sobre otros problemas frecuentes en los jóvenes: baja autoestima, fracaso académico y social, entre otros.

Actualmente muchos jóvenes manifiestan afinidad hacia una determinada estética impuesta por la sociedad contemporánea, sobre este particular, Fernández y Rodríguez (2004), han manifestado que la población joven presenta una preocupación excesiva por su cuerpo y llevan a cabo conductas de riesgo que pueden conducir al desarrollo de TAC.

La distorsión de la imagen corporal como lo revelan diversos estudios, ha demostrado que afecta particularmente al género femenino, que por motivos de índole comercial constituyen el centro en la promoción de una imagen corporal ideal, creando un culto a la delgadez (Bener y Tewfik, 2006).

Intervenciones dirigidas a elevar la autoestima corporal como medida preventiva se justifican para evitar el desarrollo de los trastornos alimentarios. En estudios realizados en adolescentes del género femenino, se pudo determinar que las que tiene niveles bajos de autoestima eran ocho veces más propensas a desarrollar trastornos alimentarios (Wardle, Williamson, Johnson y Edwards, 2006).

En ésta investigación se pretende conocer cómo se relaciona la baja autoestima por la apariencia física con la insatisfacción por la imagen corporal, en una muestra constituida por estudiantes de Ciencias de la Salud, Facultad de Medicina, Escuelas de Medicina, Enfermería y Nutrición de la Universidad de Los Andes. 


\section{MHSalud}

URL www.una.ac.cr/mhsalud

Por tanto el objetivo de este estudio es evaluar la insatisfacción por la imagen corporal y su relación con la baja estima por la apariencia física en estudiantes de la Facultad de Medicina de La Universidad de Los Andes.

\section{METODOLOGÍA}

Sujetos: constituido por 189 estudiantes (27\% masculino y $73 \%$ femenino). La edad promedio de los universitarios participantes fue de 19,58 años $\pm 1,5$ (varones 20,24 años \pm 1,76 y las mujeres 19,81 años $\pm 1,74$ ) de las carreras de la Facultad de Medicina: medicina, enfermería y nutrición, los cuales fueron seleccionados de forma intencional, ya que el objetivo del estudio tenía por finalidad conocer la presencia de factores de riesgo en una población de individuos cuya formación se oriente a la prevención de trastornos de la conducta alimentaria. Para tal fin, se seleccionó el curso único del primer año de cada carrera.

Instrumentos: Para la evaluación de la insatisfacción corporal se utilizó el Body Shape Questionnaire (BSQ) (Cuestionario sobre forma corporal) diseñado por Cooper y Taylor, (1987) y adaptado a la población española por Raich et al. (1996a). La decisión de usar este cuestionario se fundamentó en los siguientes aspectos: 1) Por ser una prueba especifica para la evaluación de insatisfacción corporal, su brevedad y su facilidad de aplicación. La confiabilidad del BSQ se calculó usando el alfa de Cronbach con el coeficiente de correlación intraclase. Los resultados confirman la estabilidad y coherencia de los presupuestos teóricos, pues su alfa fue de 0,96 con intervalo de confianza del $95 \%$ y que tuvo un límite inferior de 0,961 y un límite superior de 0,966. El factor que evalúa insatisfacción corporal tuvo un alfa de 0,95 con un intervalo de confianza del $95 \%$ y un límite inferior de 0,94 junto a un límite de 0,95 . El BSQ mostró elevada validez concurrente con otros instrumentos similares como el multidimensional Body Self-Relation Questionnaire (MBSRQ) y la subescala de insatisfacción corporal del Eating Disorders Inventory (EDI) (Raich, Torras y Figueras, 1996.)El cuestionario consta de 34 ítems referentes a la autoimagen que se evalúan mediante una escala de frecuencia de seis puntos (1. Nunca, 2. Raramente, 3. A veces, 4. A menudo, 5. Muy a menudo, 6. Siempre). De modo que el rango de la prueba es 34-204. Los factores que evalúa el BSQ son: insatisfacción corporal, miedo por engordar, sentimientos de baja autoestima por causa de la apariencia y deseo de perder peso. Para determinar la insatisfacción por la imagen corporal se sumaron los valores individuales reportados por cada participante, en cada uno de ítems que componen el cuestionario, posteriormente se determinó la media. Estableciéndose que los valores superiores a esta son considerados como "alta insatisfacción corporal" y los valores por debajo de la media se evaluaron como "baja insatisfacción corporal".

Para determinar la percepción de la imagen corporal se diseñó un cuestionario de autoregistro validado por expertos, especialistas en nutrición, médico-nutriólogos, sociólogos y psicólogos clínicos, donde se le solicitaba al participante seleccionar una opción entre cuatro alternativas diferentes de percepción de imagen corporal (Preventing and Managing the Global Epidemic (1997). (Delgado, normal, sobrepeso y obeso), y que fuese la más representativa de su condición actual, de acuerdo al peso y la talla de los participantes, para luego proceder a clasificar a los estudiantes, de acuerdo a las opciones presentadas. Posteriormente se procedió a determinar la frecuencia de la percepción de la imagen corporal de acuerdo a las variables en el estudio: sexo, condición social, carrera, etc. 


\section{MHSalud}

URL www.una.ac.cr/mhsalud

Para los fines de determinar la validez, los expertos participantes realizaron una apreciación cualitativa de cada uno de los criterios contentivos en el instrumento, lo que incluyó: claridad en la redacción de las opciones ofrecidas, pertinencia de la variable con los indicadores, relevancia del contenido y factibilidad de aplicación. Para los efectos de la validación, se aplicó el coeficiente de proporción de rangos (CPR), el cual permitió calcular la validez del contenido de cada opción, la validez del contenido de todo el instrumento y el nivel de concordancia entre jueces que validaron la prueba. Las respuestas obtenidas se sometieron al análisis cuantitativo obteniéndose una proporción de 0,9632 , lo cual indica que el instrumento presenta una excelente validez y concordancia. Para determinar la confiabilidad se utilizó el coeficiente Alpha Cronbach, el cual requiere una sola aplicación del instrumento y produce valores entre 0 y 1 . Este es un coeficiente que se utiliza para instrumentos con escalas de varias opciones como fue utilizado en la presente investigación. El coeficiente Alfa Crombach obtenido fue de 0,92, lo que determinó que existe una correlación alta entre las opciones presentadas a los participantes, esto significó que el instrumento podía ser aplicado a la muestra seleccionada con alto grado de confiabilidad.

Para determinar la condición socioeconómica de los estudiantes se aplicó el Método Graffar Modificado por Méndez y De Méndez (1994). Este método consta de 4 variables: 1. Profesión del jefe de familia, 2. Nivel de instrucción de la madre, 3. Principal fuente de ingreso de la familia y 4.Condiciones de la vivienda. Cada una de estas variables está conformada por cinco ítems, a cada ítem corresponde una ponderación decreciente del 1 al 5, la suma de los ítems determina el estrato a que pertenece la familia del estudiante investigado de acuerdo con una escala previamente diseñada.

\section{PONDERACIÓN}

$$
\begin{aligned}
& 4,5,6 \\
& 7,8,9 \\
& 10,11,12 \\
& 13,14,15,16 \\
& 17,18,19,20
\end{aligned}
$$

\section{ESTRATO SOCIAL}

Para la confirmación de la consistencia de las variables utilizadas en el Método Graffar Modificado, fue realizada una investigación por el Profesor Guillermo Laxague, Profesor de la Cátedra de Estadística y Matemática, Facultad de Ciencias Económicas y Sociales de la Universidad Central de Venezuela, de la cual las conclusiones de la investigación fueron las siguientes: a) se demostró relación entre las variables cualitativas entre sí y con las cuantitativas, b) el Método Graffar Modificado es un instrumento útil y científicamente apto para cumplir con los objetivos de estratificación social que le han sido asignado por su sencillez de implementación en el nivel práctico y por su eficacia para describir la realidad social. a) La aplicación del método en las distintas áreas de investigación ha permitido demostrar su sensibilidad y consistencia en la determinación de las distintas cuestiones investigadas y su relación generalmente determinante según estrato social (Méndez y De Méndez 1994).

Procedimiento: se seleccionaron los participantes del estudio por conglomerados de una sección del primer año de las carreras de la Facultad de Medicina de la Universidad de Los Andes. Luego se procedió a la entrega de los instrumentos para la recolección de la información socioeconómica, demográfica, antropométrica y de Imagen Corporal (delgado, normal, sobrepeso, y obesidad). 


\section{MHSalud}

URL www.una.ac.cr/mhsalud

Análisis Estadístico: A los datos se les aplicó análisis descriptivo (frecuencia, porcentajes, ANOVA de 1 solo factor para las variable independientes: género, carrera de estudio, estrato socioeconómico, y percepción de la imagen corporal) a través del paquete estadístico SPSS versión 9.0.

\section{RESULTADOS}

Tabla 1. Distribución de los participantes según área de estudio. Facultad de Medicina. Universidad de Los Andes. 2006

\begin{tabular}{|c|c|c|c|c|c|c|c|}
\hline \multicolumn{8}{|c|}{ Sexo } \\
\hline & & \multicolumn{2}{|c|}{ Masculino } & \multicolumn{2}{|c|}{ Femenino } & \multicolumn{2}{|c|}{ Total } \\
\hline & & $\mathbf{n}$ & $\%$ & $\mathbf{n}$ & $\%$ & $\mathbf{n}$ & $\%$ \\
\hline Área de & Medicina & 20 & 11 & 43 & 23 & 63 & 34 \\
\hline estudio & Nutrición & 11 & 6 & 64 & 33 & 75 & 39 \\
\hline & Enfermería & 19 & 10 & 32 & 17 & 51 & 27 \\
\hline Total & & 50 & 27 & 139 & 73 & 189 & 100 \\
\hline
\end{tabular}

En el estudio realizado participaron 189 estudiantes, 39\% se seleccionaron de la Escuela de Nutrición, 27\% de la Escuela de Enfermería y el 34\% de la Escuela de Medicina. La diferencia en los porcentajes se debe a que el número de estudiantes varía según la carrera. Es importante destacar, que para la carrera de Nutrición y Dietética, y Enfermería solo existe un curso de primer año, mientras que para el primer año de la carrera de Medicina existen 3 cursos, para efectos del estudio fue seleccionado un solo curso, el cual estuvo conformado por 63 estudiantes (Tabla 1).

La participación según género señala que un $27 \%$ son varones y $73 \%$ son mujeres. La diferencia en el número de universitarias del género femenino se debe a la estructura de la población estudiantil de la Facultad de Medicina.

En cuanto a la percepción de la imagen corporal de los estudiantes participantes, el $62.5 \%$ se perciben con una imagen corporal normal, el $25.9 \%$ se definen como delgados y el $11.6 \%$ se perciben con sobrepeso (Tabla 2).

Tabla 2. Distribución de la Percepción de Imagen Corporal

\begin{tabular}{ccc}
\hline Imagen Corporal & $\mathbf{n}$ & $\mathbf{\%}$ \\
\hline Delgado & 47 & 25,9 \\
Normal & 119 & 62,5 \\
Sobrepeso & 23 & 11,6 \\
Total & 189 & 100
\end{tabular}

Respecto a la percepción de la imagen corporal y género, en la tabla 3 se observaron diferencias en el porcentaje, donde existe una mayor percepción de imagen corporal caracterizada por el sobrepeso y la obesidad en las mujeres. A través de la prueba de Chi cuadrado se pudo determinar una relación estadísticamente significativa entre el género y la imagen corporal percibida $\chi^{2}(2, \mathrm{~N}=189)=9,586, \mathrm{p}=0,008$. Para efectos de este estudio se detectó un caso con obesidad, femenino de la clase media alta, que fue asimilado por la categoría de sobrepeso pues solo se trataba de un sujeto.

Tabla 3. Distribución según el tipo de percepción de la Imagen Corporal y Género 


\section{MHSalud}

URL www.una.ac.cr/mhsalud

\begin{tabular}{ccccccccc}
\hline Sexo & \multicolumn{2}{c}{ Delgado } & \multicolumn{2}{c}{ Normal } & \multicolumn{2}{c}{ Sobrepeso } & \multicolumn{2}{c}{ Total } \\
\hline Masculino & $\mathrm{n}$ & $\%$ & $\mathrm{n}$ & $\%$ & $\mathrm{n}$ & $\%$ & $\mathrm{n}$ & $\%$ \\
& 15 & 6.9 & 29 & 14.3 & 6 & 3.3 & 50 & 24.5 \\
Femenino & 32 & 19 & 90 & 48.1 & 17 & 7.9 & 139 & 75.5 \\
Total & 47 & 25.9 & 119 & 62.4 & 23 & 11.2 & 189 & 100 \\
\hline
\end{tabular}

En relación con la percepción de la imagen corporal según el área de estudio, se observó mayor frecuencia de imagen corporal de sobrepeso en los universitarios que cursan la carrera de Nutrición y Dietética (8\%). El porcentaje de estudiantes que se autoperciben como delgados fue similar en las tres escuelas (Tabla 4).

Tabla 4. Percepción de la Imagen Corporal según el Área de Estudio

\begin{tabular}{ccccccccc}
\hline Institución & \multicolumn{2}{c}{ Delgado } & \multicolumn{2}{c}{ Normal } & \multicolumn{2}{c}{ Sobrepeso } & \multicolumn{2}{c}{ Total } \\
\hline Nutrición y & $\mathrm{n}$ & $\%$ & $\mathrm{n}$ & $\%$ & $\mathrm{n}$ & $\%$ & $\mathrm{n}$ & $\%$ \\
Dietética & 15 & 8.0 & 45 & 24.0 & 15 & 8.0 & 75 & 40.0 \\
Enfermería & 15 & 8.0 & 32 & 16.5 & 3 & 1.5 & 51 & 26.0 \\
Medicina & 17 & 9.0 & 42 & 22.0 & 5 & 3.0 & 63 & 34.0 \\
TOTAL & 47 & 25.0 & 119 & 63.5 & 23 & 12.5 & 189 & 100 \\
\hline
\end{tabular}

Con respecto al estrato social de los universitarios y la imagen corporal, se observó que el Estrato III registró los porcentajes más elevados con la percepción de normal $(37,6 \%)$, delgado $(14,8 \%)$ y sobrepeso $(6,9 \%)$, reportándose el único caso de autopercepción de obesidad en el referido estrato social (Tabla 5).

Tabla 5. Distribución según el tipo de percepción de la imagen corporal y estrato socioeconómico

\begin{tabular}{|c|c|c|c|c|c|c|c|c|c|c|}
\hline \multicolumn{11}{|c|}{ Condición Socioeconómica } \\
\hline \multirow{2}{*}{$\begin{array}{l}\text { Imagen } \\
\text { Corporal }\end{array}$} & \multicolumn{2}{|c|}{ Estrato social II } & \multicolumn{2}{|c|}{ Estrato Social III } & \multicolumn{2}{|c|}{ Estrato Social IV } & \multicolumn{2}{|c|}{ Estrato V } & \multicolumn{2}{|c|}{ Total } \\
\hline & $\mathrm{n}$ & $\%$ & $\mathrm{n}$ & $\%$ & $\mathrm{n}$ & $\%$ & $\mathrm{n}$ & $\%$ & $\mathrm{n}$ & $\%$ \\
\hline Delgado & 8 & 4.2 & 28 & 14.8 & 12 & 6.3 & 1 & 0.5 & 47 & 25.0 \\
\hline Normal & 24 & 12.7 & 71 & 37.6 & 20 & 10.6 & -- & -- & 119 & 63.5 \\
\hline Sobrepeso & 3 & 1.6 & 13 & 6.9 & 6 & 3.1 & 3 & 1.7 & 22 & 12.0 \\
\hline Total & 35 & 18.5 & 112 & 59.3 & 38 & 20.0 & 4 & 2.2 & 189 & 100 \\
\hline
\end{tabular}

Una vez descritos los valores que expresan la imagen corporal de los estudiantes, corresponde analizar las cuestiones referidas a la insatisfacción relacionada con esa imagen; el género, el área de estudio y estrato socioeconómico.

\section{INSATISFACCIÓN POR LA IMAGEN CORPORAL}

\section{Insatisfacción con la Imagen Corporal y Percepción de la Imagen Corporal}

Obtenida la media de la insatisfacción corporal de los participantes, se observa que los sujetos con percepción de sobrepeso registraron niveles de insatisfacción superiores a la media obtenida para el total de estudiantes ( $\bar{X} 120 \pm \mathrm{DE}=16.77$ puntos $)$; los estudiantes con percepción normal obtuvieron puntajes aproximados a esa media y los que se percibieron como delgados alcanzaron valores por debajo de la misma ( $\bar{X} 58.24 \pm \mathrm{DE}=12.49$ puntos$)$. Las diferencias entre las medias de insatisfacción según el tipo de imagen corporal percibida son estadísticamente significativas $(\mathrm{F}=11.236 ; \mathrm{p}<0.001)$. Por tanto, es evidente que el nivel de insatisfacción de los participantes puede estar asociado con su imagen corporal percibida. 


\section{MHSalud}

URL www.una.ac.cr/mhsalud

\section{Insatisfacción con la Imagen Corporal y Género}

En cuanto a la insatisfacción con la imagen corporal relacionada con el género, se encontró que la población femenina reportó una media de insatisfacción $(\bar{X} 70.01 \pm \mathrm{DE} 15.77$ puntos) significativamente más alta $(\mathrm{F}=10.23 ; \mathrm{p}=0.002)$ en relación con el género masculino ( $\bar{X} 59.48 \pm$ DE 15.79 puntos). Esto evidencia que la insatisfacción con la imagen corporal se asocia con el género y por tanto, debería analizarse desde esta perceptiva. Las féminas, por tanto, presentaron mayor preocupación por la imagen.

\section{Insatisfacción y Área de Estudio}

En cuanto a la comparación de insatisfacción con la imagen corporal, por área de estudio, no se encontraron diferencias estadísticamente significativas $(\mathrm{F}=0.085 ; \mathrm{p}=0.918)$.

En cuanto a la insatisfacción con la imagen corporal comparada según el estrato socioeconómico, no se encontraron diferencias estadísticamente significativas $(\mathrm{F}=0.665$; $\mathrm{p}=0.575$ ). Lo cual demuestra que las diferencias en la insatisfacción no se asocian a la variable condición social de los participantes.

\section{BAJA AUTOESTIMA POR LA APARIENCIA FÍSICA}

\section{Baja autoestima por la Apariencia Física y Percepción de la Imagen Corporal}

En relación con la baja autoestima por la apariencia física, una vez calculada la media $(\bar{X}$ $9.69 \pm$ DE 4.14), se observa que los universitarios con percepción de sobrepeso ( $\bar{X} 11.77 \pm$ DE 6.89) reportaron niveles de baja autoestima por la apariencia física más altos que aquellos que reportaron percepción de delgado ( $\bar{X} 8.36 \pm$ DE 2.24$)$ y normal ( $\bar{X} 10.17 \pm$ DE 3.80). Los valores de la prueba Post hoc de Duncan para baja autoestima por la apariencia física, se presentan en la Tabla 6.

Tabla 6. Valores prueba Post hoc de Duncan para baja autoestima por la apariencia física

\begin{tabular}{lccc}
\hline $\begin{array}{c}\text { Imagen corporal actual } \\
\text { percibida }\end{array}$ & $\mathbf{n}$ & $\begin{array}{c}\mathbf{1} \\
\text { (alfa 0,05) }\end{array}$ & $\begin{array}{c}\mathbf{2} \\
\text { (alfa 0,05) }\end{array}$ \\
\hline Delgado & 33 & 8,3636 & 10,1798 \\
Normal & 89 & 10,1798 & 11,7778 \\
Sobrepeso & 18 & 0,078 & 0,121 \\
Significancia & & & \\
\hline
\end{tabular}

\section{Baja Autoestima por la Apariencia Física y Género}

Acerca de la baja autoestima y el género, los valores para la población femenina son apreciablemente elevados ( $\bar{X} 10.36 \pm$ DE 4.42) con respecto a la media calculada ( $\bar{X} 9.95 \pm$ DE 4.14) y también con los valores de la población masculina ( $\bar{X} 8.37 \pm \mathrm{DE} 2.33)$. Las diferencias encontradas en la baja autoestima por la apariencia física, según el género, son estadísticamente significativas $(\mathrm{F}=5,45 ; \mathrm{p}=0.021)$.

\section{Baja Autoestima por Apariencia Física y Área de Estudio}

Los participantes de las escuelas de Enfermería ( $\bar{X} 10.26 \pm$ DE 4.9) y Medicina ( $\bar{X} 10.66 \pm$ DE 3.15) presentaron registros de baja autoestima por la apariencia física más altos que los obtenidos por los estudiantes de nutrición ( $\bar{X} 9.61 \pm$ DE 3.70). Cabe destacar que las diferencias encontradas en baja autoestima por la apariencia física, según el área de estudio, no son estadísticamente significativas, por lo que la baja autoestima por la apariencia física no está asociada a la carrera cursada por los participantes ( $\mathrm{F}=0.606 ; \mathrm{P}=0.547)$. 


\section{MHSalud}

URL www.una.ac.cr/mhsalud

\section{Baja Autoestima por Apariencia y Condición Social}

En relación con la baja autoestima por la apariencia física, según la condición social, se puede apreciar descriptivamente, que los participantes de los estratos sociales media, baja y obrera, registraron niveles de baja autoestima por la apariencia física superiores a los de los sujetos pertenecientes a los estratos sociales media alta y media. Pero no se encontraron diferencias significativas en los valores reportados para cada clase social $(F=1.54 ; p=0.206)$.

\section{DISCUSIÓN}

Los resultados obtenidos ponen de manifiesto diferencias por género, en lo que respecta a la percepción de la imagen corporal entre los universitarios participantes. Al respecto, existe un mayor número de participantes del género femenino con percepción de sobrepeso. La percepción de una imagen con estos rasgos crea un mayor descontento por el aspecto fisiológico global en las mujeres que en los hombres, mientras que ser demasiado delgado se relaciona con una pobre valoración del autoconcepto físico particularmente en los hombres Estos resultados son comparables a los obtenidos en investigaciones con los mismos propósitos (Davison y McCabe, 2006). También para este aspecto de la distorsión de la imagen corporal, se encuentran coincidencias con los estudios sobre valoración de la percepción de la imagen corporal mediante modelos anatómicos (Montero, Morales, Carvajal 2004), donde se observan claramente diferencias en la percepción de la imagen corporal en función del género; las mujeres se perciben más obesas de lo que en realidad son, mientras que los hombres se perciben en forma más real. Las diferencias anteriores obtenidas por el género y su coincidencia con las investigaciones citadas, reflejan la existencia de mayor insatisfacción con su propio cuerpo por parte de las féminas (Furnham, Badmin y Sneade, 2002).

Según Moore (1993), los individuos que están inconforme con su imagen corporal presentan mayor riesgo en prácticas de control de peso potencialmente perjudiciales, tales como; hacer dietas, inducción del vómito, uso de diuréticos, laxantes y uso de anorexigenos. La insatisfacción con la imagen corporal y su relación con las áreas de estudio y la condición social de los participantes se estableció, en primer lugar, que a excepción de un valor superior a la media calculada en medicina, no se observaron diferencias estadísticamente significativas entre los niveles de insatisfacción encontrados y las escuelas evaluadas; en segundo lugar, a pesar de reportarse que los estudiantes de procedencia media baja, tienen valores de insatisfacción superiores a la media, no se observaron diferencias estadísticamente significativas en relación con la insatisfacción reportada para el resto de los participantes. Tal hecho demostraría que las diferencias en la insatisfacción no se asocian a la variable condición social de los participantes. En esto hay una relativa diferencia con los estudios que han relacionado la influencia de la clase social con los niveles de insatisfacción con la imagen corporal, al respecto.

Ozmen, Ozmen Ergin y Cakmakci (2007), han informado sobre el factor predictivo que tiene la condición social en la satisfacción con la imagen corporal, donde las mujeres de estratos socioeconómicos más alto presentaron niveles más elevados de insatisfacción con la imagen percibida que aquellas pertenecientes a estratos socioeconómicos más bajos.

En cuanto a la autoestima por la apariencia física, se encontró que el nivel de baja autoestima por la apariencia física es estadísticamente significativo en relación con la percepción de la imagen. En la población estudiada se observó que los participantes con autopercepción de obesos y sobrepeso, tienen niveles de bajo autoestima más altos que 


\section{MHSalud}

URL www.una.ac.cr/mhsalud

aquellos que se perciben delgados y normales. Estos resultados coinciden con aquellos estudios que señalan como la baja autoestima por la apariencia física se convierte en una consecuencia asociada a la pobre imagen corporal (Bener y Tewfik, 2006). También dejan en evidencia que la población femenina tiene valores apreciablemente elevados de baja autoestima con respecto a la masculina. Igualmente es observable que la baja autoestima por la apariencia no está asociada a la carrera cursada por los participantes, ya que si bien los de enfermería y medicina presentan baja autoestima más elevada que los de nutrición, las diferencias no son estadísticamente significativas. Esto se observó también en los resultados para la condición social, los cuales no expresan diferencias significativas en los valores reportados para cada clase social.

Un enfoque más integral para interpretar los resultados, va a considerar a la autoestima por la apariencia física, en la población investigada, como un hecho institucional dinamizado por factores sociales condicionantes. Esto se afirma, porque precisamente, cada uno de los valores obtenidos para la baja autoestima, son constituyentes de las relaciones sociales e interpersonales, que influyen para la conformación de la autoestima por la apariencia física.

Finalmente los resultados obtenidos permiten sugerir sobre la relación que existe entre la insatisfacción y la baja autoestima por la apariencia física, donde el rechazo por la propia imagen corporal causada por la percepción distorsionada o no de la misma, podría afectar la estima por la apariencia física. El presente estudio confirma algunos hallazgos realizados en otras latitudes donde la baja autoestima por la apariencia física se encuentra relacionada con los niveles de insatisfacción por su imagen corporal. Ozmen, Ozmen y Ergin (2007). Según el referido estudio los resultados indican que el bienestar psicológico de los adolescentes se relaciona más a la satisfacción con la imagen corporal que con la imagen corporal percibida.

Por consiguiente, el grupo participante debe ser analizado en su autoestima por la apariencia física, como una expresión en el ámbito institucional, de una problemática presente en la población joven venezolana, así como en la diversidad de países, unos llamados desarrollados y otros en vías de alcanzarlo. De acuerdo a los resultados obtenidos, la población investigada es una muestra representativa de los problemas relacionados con la imagen corporal y a la insatisfacción con la misma. Es importante señalar, para la caracterización de los participantes, que su edad promedio los aproxima a los grupos adolescentes y a sus problemas característicos. Este hecho puede relacionarse con el proceso de ingreso a la Universidad de los Andes, ya que en su matrícula se incorporan porciones importantes de esos grupos de edad, provenientes también de esta región y del Estado Mérida. Al respecto, es pertinente señalar que para los resultados de esta investigación, pueden considerarse como significativos los hallazgos del estudio realizado por López (2004), referidas a la influencia de los factores sociodemográficos sobre la Imagen corporal en adolescentes en la ciudad de Mérida.

El grupo investigado, como representativo de la problemática mencionada y en su condición de expresión institucional, al formar parte de las relaciones interpersonales presentes en el nivel familiar, institucional y social en general, de acuerdo a los resultados obtenidos, muestra cómo cada uno de sus participantes hacen una evaluación cognitiva de su propio cuerpo, y por lo tanto, de su apariencia. De los resultados se infiere que siendo un grupo integrado en un $76 \%$ por el sexo femenino; de procedencia social de clase media y media alta en un $77 \%$ y con una imagen corporal de delgado y normal de más de $88 \%$, sus participantes experimentan niveles de inquietud o preocupación en parámetros que pueden considerarse normales o no excesivos en tal evaluación cognitiva; lo cual puede traducirse 


\section{MHSalud}

URL www.una.ac.cr/mhsalud

en una incidencia relativa para la autoestima tanto en lo grupal como en lo individual. Al respecto, es demostrativo cómo los valores de la baja autoestima, se expresan en los niveles relativamente homogéneos en todos los participantes, sin obviar las excepciones impuestas por las razones del sexo y la condición social.

Esos niveles de homogeneidad no excluyen, las influencias que experimenta el grupo, justamente por la propia dinámica de las relaciones sociales e interpersonales de los cuales forma parte; entre otros, su exposición permanente a los mensajes de los medios de comunicación, cuando estos difunden los llamados ideales físicos; la existencia de la comparación social en lo interpersonal, manifestándose en el miedo por ganar peso corporal y en las expectativas para el mejoramiento de la apariencia, etc. Es obvio que estas cuestiones están presentes como motivaciones en este grupo de estudiantes de Ciencias de la Salud.

Los resultados obtenidos en la presente investigación, acerca de los grados de preocupación con la imagen corporal y los problemas que se le vinculan, en los estudiantes de Ciencias de la Salud, confirman las conocidas consecuencias para la insatisfacción corporal ligada a la baja autoestima; sobre los trastornos de la conducta alimentaría, etc., como factores de riesgo que conducen a la anorexia y la bulimia. También ilustran sobre las expectativas que desarrollan acerca del mejoramiento de la figura, incluso, con las experiencias de la cirugía estética.

Para interpretar los resultados, al asumir que están relacionados con factores socioculturales; también servirían para analizar los grados de diferenciación existentes entre los estudiantes participantes de Ciencias de la Salud. Esto puede significar, que independientemente de la condición para todos como actores participantes en el campo de la salud, los de medicina y enfermería son visualizados ocupándose solamente de lo orgánico-funcional, mientras los de nutrición estarían más directamente ocupándose, como actores también participantes, de las variables que miden la presión social bajo la óptica de lo orgánico-corporal.

Finalmente, como una interpretación de esta relativa condición diferenciada para los estudiantes de nutrición, como participantes de esta investigación, debe afirmarse que teóricamente experimentan una propensión más acentuada para elevar su autoestima por la apariencia.

\section{CONCLUSIONES}

Los hallazgos obtenidos en el presente estudio son reveladores de la presencia de factores de riesgo relacionados con los trastornos de la conducta alimentaria. A pesar de que el objetivo del estudio fue determinar o diagnosticar la existencia de Trastornos de la Conducta Alimentaria, se puede considerar que una parte de los individuos que participaron en el estudio se encuentran en riesgo de padecer o sufrir dichas patologías. Estos resultados deben ser tomados en cuenta por los entes gubernamentales con el propósito de elaborar programas preventivos para mejorar la salud mental de los jóvenes. Es importante destacar que las intervenciones deben considerar a los grupos poblacionales con mayor riesgo, particularmente desde la infancia. La literatura hace referencia que los trastornos de la alimentación no establecen diferencia en cuanto a la condición social, raza etc. Se han considerado un verdadero problema de salud pública, en virtud, de las consecuencias que se asocian en ellas. Entre las que se pueden citar están los altos costos del tratamiento y los graves problemas de tipo psicológico de quien la padece. 


\section{MHSalud \\ URL www.una.ac.cr/mhsalud}

En Latinoamérica, particularmente en Venezuela apenas se hacen intentos por conocer los aspectos epidemiológicos relacionados con el tema, sin considerar la urgente necesidad de que las actividades preventivas tienen un carácter prioritario, al respecto, es muy poco o nada lo que se ha hecho sobre el particular, ya que no se cuenta con la experiencia y el personal especializado para dar inicio a la toma de conciencia de este nuevo flagelo que la sociedades modernas han creado.

\section{RECOMENDACIONES}

Teniendo presente los resultados obtenidos de la investigación, sobre la existencia entre la población universitaria estudiada, de una problemática que se manifiesta, entre otras cuestiones, por su preocupación por la imagen corporal y los grados de trastornos que ocasionan. Los resultados sugieren la conformación de un equipo multidisciplinario con la finalidad de evaluar e implementar medidas preventivas que tengan como objetivos incluir el impacto de la distorsión de la imagen corporal de los universitarios, lo cual se constituye en una magnifica oportunidad para los estudiantes del área de nutrición y dietética de participar en actividades de extensión de las ya mencionadas para promover el bienestar de la población estudiantil.

Los hallazgos encontrados tienen implicaciones para la prevención y tratamiento de los trastornos psicológicos de los adolescentes con alteraciones distorsión de su imagen corporal, particularmente aquellos que manifiestan niveles de insatisfacción con su imagen más alta.

Es importante destacar que las actividades preventivas deben tomar en consideración como población objetivo, a los padres, particularmente en aquellos casos donde las alteraciones por la imagen corporal puedan presentarse en edades más temprana con la finalidad de minimizar el impacto en la autoestima.

En virtud, del papel que juegan los medios de comunicación en el desarrollo de las actitudes relacionadas con la imagen corporal, es necesario que se implemente políticas que tengan como objetivo la promoción de hábitos alimenticios saludables y la práctica de ejercicio con el propósito de prevenir la obesidad en los grupos de mayor vulnerabilidad.

\section{REFERENCIAS}

American Psychiatric Association. (2000). Diagnostic and Statistical Manual of Mental Disorders, DSM-IV-TR. Arlington, VA.: American Psychiatric Press.

Banfield, S. S. y McCabe, M. P. (2002). An evaluation of the construct of body image. Adolescence, 37, 373-393.

Bener, A. Tewfik I. (2006). Prevalence of overweight, obesity, and associated psychological problems in Qatari's female population. Obesity Reviews 7 (2), 139-145.

Bonilla, P., \& Martínez, L. (2001). Adolescencia y género: Imagen corporal y trastornos de la alimentación. Cuadernos Mujer Salud, 6, 156-164. 


\section{MHSalud}

URL www.una.ac.cr/mhsalud

Brown, T. A., Cash, T. F. y Mikulka, P. J. (1990). Attitudinal body-image assessment: factor analysis of the Body-Self Relations Questionnaire. Journal of Personality Assessment, 55,135-144.

Buddeberg, B., Klaghofer, F, y Reed, V. (1999). Associations between body weight, psychiatric disorders and body image in female adolescents. Psychotherapy and Psychosomatics, 68, 325-338.

Cooper, P. y Taylor, M. (1987). The development and validation of the body shape Questionnaire. International Journal of Eating Disorders. 6; 485-494.

Davison, T.E. y McCabe, M.P. (2006). Adolescent body image and psychosocial functioning .J Soc Psycho, 146 (1):15-30.

Fernández, L. y Rodríguez, Y. (2003). Evaluación de los hábitos alimentarios e Imagen Corporal en Estudiantes Universitarios sin trastornos alimentarios. International Journal of Clinical and Health Psychology, 3, (1), 23-33.

Furnham A, Badmin N, Snead I: (2002). Body image dissatisfaction: gender differences in eating attitudes, self-esteem, and reason for exercise. J Psychol, 136 (6): 581-96.

López, P. J. (2004). Influencia de factores Sociodemográficos sobre la imagen corporal en adolescentes. Universidad de Los Andes. Facultad de Medicina. Escuela de Nutrición y Dietética. Manuscrito no publicado.

Méndez, H. y De Méndez, M. (1994). Sociedad y Estratificación. Método Graffar-Méndez Castellano. Venezuela: Ed. FUNDACREDESA.

Montero, P., Morales, E. y Carvajal, A. (2004). Valoración de la percepción de la Imagen Corporal mediante modelos anatómicos. Antropo, 8, 107-116.

Moore DC (1993): Body image and eating behavior in adolescents. J Am Coll. Nutr, 12 (5), 5005.

Preventing and Managing the Global Epidemic (1997). Report of WHO Consultation on obesity. Ginebra WHO, 3-5.

Ozmen D, Ozmen E, Ergin D, Cakmakci A. (2007). The association of self-esteem, depression and body satisfaction among Turkish adolescent. Public Health, 7, 80.

Raich, R., Torras, J., y Figueras, M. (1996). Estudio de la Imagen Corporal y su relación con el deporte en una muestra de estudiantes universitarios. Análisis y Modificación de Conducta. 22, 604-624.

Raich, R. M, Mora, M., Soler, A., Avila, C., Clos, I. y Zapater, L. (1996a). Adaptación de un instrumento de evaluación de la insatisfacción corporal. Clínica y Salud, 7, 5166.

Strauss R, Pollck H.(2003). Social Marginalization of overweight children. Arch Pediatric Adolescent Med, 157, 746-752. 


\section{MHSalud}

URL www.una.ac.cr/mhsalud

Wardle J, Williamson S, Johnson F, Edwards C. (2006). Depression in adolescent obesity: Cultural moderators of the depressive symptoms. Int J Obes (Lond), 30, 634-43.

Xie B, Liu C, Chou CP, Xia J, Spruijt-Metz D, Gong J. (2003). Weight perception and psychology factors in adolescents. J Adolesc Health, 33, 202-210.

Fecha de recepción del artículo: 9 de noviembre del 2007.

Fecha de aceptación del artículo: 31 de marzo del 2007.

Fecha de publicación del artículo: 31 de julio del 2008. 